\title{
Physical activity energy expenditure measured using a portable telemetric device in comparison with a mass spectrometer
}

\author{
C Maiolo, G Melchiorri, L lacopino, S Masala, A De Lorenzo
}

Br J Sports Med 2003;37:445-447

See end of article for authors' affiliations

.....................

Correspondence to:

Professor De Lorenzo,

Division of Human

Nutrition, Department of

Neurosciences, Faculty of

Medicine and Surgery,

University of Rome "Tor

Vergata", Via Montpellier,

1, 00133 Rome, Italy;

delorenzo@uniroma2.it

Accepted

20 October 2002

\begin{abstract}
Background: Several studies have evaluated the accuracy of portable metabolic gas analysis systems, which measure physical activity energy expenditure. Since the Cosmed K2 telemetric system, two K4 devices (RQ and b2) have been developed.

Objective: To compare measurements using the Cosmed K4 RQ with measurements by a traditional method (mass spectrometer) for a sample of healthy subjects performing physical exercise of various intensities.

Methods: Nine healthy male footballers (mean (SD) age 18.3 (2.2) years) performed an incremental exercise running test from $8 \mathrm{~km} / \mathrm{h}$ to exhaustion, with an increase of $2 \mathrm{~km} / \mathrm{h}$ for each level of the test protocol. The expired air was collected simultaneously using the Cosmed K4 RQ and Airspec QP9000 mass spectrometer. Oxygen consumption $\left(\mathrm{VO}_{2}\right)$ and carbon dioxide production $\left(\mathrm{V}_{\mathrm{CO}_{2}}\right)$ were measured at rest and at each exercise intensity.

Results: No significant differences were found between $\mathrm{VO}_{2}$ and $\mathrm{VcO}_{2}$ measured by the two instruments, at rest or during exercise.

Conclusions: The application of the Cosmed $\mathrm{K} 4 \mathrm{RQ}$ system for $\mathrm{VO}_{2}$ and $\mathrm{V}_{\mathrm{CO}_{2}}$ measurements at rest and during both submaximal and maximal exercise may be of interest to sport physiologists, nutritionists, and doctors.
\end{abstract}

T he metabolic cost of different physical activities, such as walking, running, working, and other sporting activities, in addition to the resting metabolic rate is useful for assessing the energy expenditure (EE) of a subject during a representative day. ${ }^{1}$ Whereas resting metabolic rate can usually be measured in clinical settings, EE during exercise is difficult to measure using indirect calorimetry. ${ }^{2}$

Over the last 30 years, technical innovations such as portable systems for metabolic gas analysis have allowed metabolic measurements of physical EE to be made in the office, field, and home settings. It has been suggested that these automated systems would increase the accuracy and reliability of exercise tests. ${ }^{3}$ However, it is important to check the accuracy and reliability of the flow and gas measurements. The Douglas bag technique, which is often used as reference method, has been shown to be valid and accurate, ${ }^{4}$ but the use of this system has some difficulties-for example, diffusion of gases through the bag. ${ }^{56}$ The Cosmed K2 portable device, which measures only oxygen uptake, has been shown to be accurate at rest ${ }^{7}$ and at both submaximal and maximal exercise levels. ${ }^{89}$ Successively, two Cosmed K4 devices (RQ and b2) containing a $\mathrm{CO}_{2}$ electrode have been developed. Only a few studies have examined the validity and reliability of these systems compared with a standard technique. ${ }^{60-12}$ We have previously reported an in vitro experimental study to validate the use of Cosmed K4 RQ (K4 $\mathrm{RQ}$ ) for measuring flow rate and gas concentrations. ${ }^{13}$

The aim of this study was to compare in vivo measurements of the K4 RQ with measurements obtained using a traditional mass spectrometer for a sample of healthy subjects performing physical exercise of various intensities. We compare the accuracy and validity of $\mathrm{VO}_{2}$ and $\mathrm{VCO}_{2}$ measurements between the two systems using simultaneous data collection.

\section{METHODS}

\section{Subjects}

Nine healthy male volunteer footballers (non-professional athletes) were selected randomly to participate in the study.
Mean (SD) age, body weight, and height were 18.3 (2.2) years, $67.3(4.6) \mathrm{kg}$, and 1.77 (0.57) m respectively. Each participant gave his written informed consent. The ethics committee of the University of Rome "Tor Vergata" approved the study protocol.

\section{Instrumentation}

The K4 RQ, as described previously, ${ }^{13}$ consists of three main components: a system to sample the expired air (mask and flowmeter); a portable transmitting unit (which measures 170 $\times 90 \times 50 \mathrm{~mm}$ and weighs $550 \mathrm{~g}$ ), fixed to a chest harness; and a receiving unit. It uses a Cosmed certified oxygen analyser (range 7-24\%) and an infrared non-dispersive thermostatically controlled carbon dioxide analyser (range 0.0-8\%). Before each test protocol, the instrument was calibrated against inspired air assumed to have a fractional oxygen concentration of $20.9 \%$ and a fractional carbon monoxide concentration of $0.03 \%$, as described elsewhere. ${ }^{10}{ }^{13}$ The flowmeter uses a bidirectional digital turbine and opto-electric reader, and, according to the manufacturer, has a linear response in the ventilation range $0.0-300$ litres $/ \mathrm{min}$. The turbine flowmeter was calibrated using a 3 litre syringe at the beginning of the test protocol. This turbine was found to be accurate as a flowmeter. ${ }^{13}{ }^{14}$ According to the technical description provided by the manufacturer, the $18 \mathrm{~mm}$ section turbine was used for measurements at rest, and the $28 \mathrm{~mm}$ section turbine was used for measurements during physical exercise. Oxygen consumption $\left(\mathrm{VO}_{2}\right)$ and carbon dioxide production $\left(\mathrm{VCO}_{2}\right)$ were sampled every 15 seconds, and their values were measured at rest and at various exercise intensities $(8,10,12,14,16$, and $18 \mathrm{~km} / \mathrm{h})$.

The Airspec QP9000 (QP9000) is a quadrupole mass spectrometer housed in a robust high vacuum system. It is specifically designed for respiratory gas analysis and consists of a custom built dual microprocessor based controller (digital unit) connected to a personal computer. Levels of inspired and 
Table 1 Oxygen consumption $\left(\mathrm{VO}_{2}\right.$, litres/min), carbon dioxide production $\left(\mathrm{V}_{\mathrm{CO}_{2}}\right.$, litres/min), and respiratory exchange ratio (RER) measured with a Cosmed K4 RQ and an Airspec QP9000 mass spectrometer at rest and at different exercise levels (8, $10,12,14,16$, and $18 \mathrm{~km} / \mathrm{h}$ ) in nine male footballers

\begin{tabular}{|c|c|c|c|c|c|c|}
\hline \multirow{2}{*}{$\begin{array}{l}\text { Exercise } \\
\text { level }\end{array}$} & \multicolumn{3}{|c|}{ Cosmed K4 RQ } & \multicolumn{3}{|c|}{ Airspec QP9000 } \\
\hline & $\mathrm{VO}_{2}$ & $\mathrm{VCO}_{2}$ & RER & $\mathrm{Vo}_{2}$ & $\mathrm{VCO}_{2}$ & RER \\
\hline Rest & $0.27(0.10)$ & $0.20(0.08)$ & $0.74(0.08)$ & $0.29(0.16)$ & $0.21(0.09)$ & $0.72(0.07)$ \\
\hline 8 & $2.26(0.31)$ & $1.87(0.23)$ & $0.83(0.05)$ & $2.15(0.37)$ & $1.85(0.29)$ & $0.86(0.06)$ \\
\hline 10 & $2.72(0.33)$ & $2.38(0.21)$ & $0.88(0.04)$ & $2.61(0.34)$ & $2.29(0.28)$ & $0.88(0.05)$ \\
\hline 12 & $3.12(0.24)$ & $2.82(0.17)$ & $0.90(0.06)$ & $3.06(0.26)$ & $2.78(0.29)$ & $0.91(0.06)$ \\
\hline 14 & $3.39(0.32)$ & $3.34(0.21)$ & $0.99(0.12)$ & $3.21(0.42)$ & $3.04(0.33)$ & $0.95(0.07)$ \\
\hline 16 & $3.64(0.19)$ & $3.63(0.37)$ & $1.00(0.11)$ & $3.59(0.31)$ & $3.52(0.33)$ & 0.98 (0.09) \\
\hline 18 & $3.72(0.18)$ & 4.05 (0.39) & 1.09 (0.09) & $3.80(0.36)$ & $4.03(0.46)$ & $1.06(0.07)$ \\
\hline
\end{tabular}

expired $\mathrm{O}_{2}$ and $\mathrm{CO}_{2}$ were determined at rest and at each level of the exercise protocol using this instrument.

\section{Test protocol}

The test protocol was conducted using the same tapis roulant (Technogym, Pesaro, Italy) for all participants, who performed an incremental exercise running test from $8 \mathrm{~km} / \mathrm{h}$ to exhaustion, with an increase of $2 \mathrm{~km} / \mathrm{h}$ for each level of the test protocol. The duration of each running test was set at six minutes. Only the mean values of measurements of the last two minutes were considered for further analysis. Before each exercise level, the participants rested for two minutes. The expired air was collected simultaneously for every subject using the K4 RQ and QP9000, which were disposed in parallel with a special small connector of low resistance respiratory value. Ventilation levels for $\mathrm{VO}_{2}$ and $\mathrm{VCO}_{2}$ calculation were measured with the K4 RQ.

\section{Data analysis}

Statistical comparisons between $\mathrm{VO}_{2}$ and $\mathrm{VCO}_{2}$ measured using the K4 RQ and QP9000 were made with an SPSS software package. Planned contrasts to limit comparisons between instruments for each condition were used. Mean values for measurements were analysed using Student's paired $t$ test. A significance level of $\mathrm{p}<0.05$ was chosen for all comparisons.

\section{RESULTS}

Table 1 gives the mean values for $\mathrm{VO}_{2}, \mathrm{VCO}_{2}$, and respiratory exchange ratio measured using the K4 RQ and QP9000 at rest and at different exercise levels $(8,10,12,14,16$, and $18 \mathrm{~km} / \mathrm{h})$. No significant differences were found between measurements using the two instruments either at rest or at any exercise level. Mean (SD) ventilation ranged from 8.8 (1.18) litres/min at rest to $116.50(11.50)$ litres/min for the highest exercise level.

\section{DISCUSSION}

There are many reports of EE at rest and various workloads. ${ }^{35-17}$ Each athlete has unique energy requirements, which can be predicted by estimating resting metabolic rate and activity levels. ${ }^{18}$ Many studies have also evaluated the accuracy of portable metabolic gas analysis systems, which measure physical activity EE. ${ }^{6-14}$ An indirect calorimeter with a telemetric system is a convenient, accessible, and highly accurate instrument for measuring energy requirements of athletes. We have found that the portable metabolic system Cosmed K4 RQ is practical and precise for the determination of EE cost in athletes. Our study shows that data obtained with the K4 RQ portable calorimeter were not significantly different from those obtained with the Airspec QP9000 mass spectrometer. We compared $\mathrm{VO}_{2}$ and $\mathrm{VCO}_{2}$ measurements using simultaneous data collection. McLaughlin et $a l^{11}$ found no significant difference between $\mathrm{VO}_{2}$ at rest and at maximal workloads (250 W) measured using a K4 RQ and the Douglas bag method. However, the subjects were tested on consecutive days to avoid interference between the two methods, which may result in differences in $\mathrm{VO}_{2}$ caused by day to day variation. One advantage of our study is that participants were tested on the same day. $\mathrm{VO}_{2}$ and $\mathrm{VCO}_{2}$ measured with the K4 RQ were not significantly different from those measured with the QP9000 both at rest and at every exercise level.

The results confirm the validity of the K4 RQ system for gas measurements in the same subject at rest and during both submaximal and maximal exercise, suggesting its possible use for measuring EE over a wide range of exercise intensities. It may prove to be a valuable tool for optimising nutritional assessment of athletes. Measurement of EE during exercise may estimate the total EE compared with calculated predictions for nutritional needs. Application of the K4 RQ portable indirect calorimeter may be of interest to sport physiologists, nutritionists, and doctors.

\section{ACKNOWLEDGEMENTS}

We thank Dr Marcello Faina for invaluable help during the preparation of the article and Dr Ehab I Mohamed for discussion and help during the revision of the manuscript.

\section{Authors' affiliations}

C Maiolo, G Melchiorri, L lacopino, A De Lorenzo, Division of Human Nutrition, Faculty of Medicine and Surgery, University of Rome "Tor Vergata", Rome, Italy

S Masala, Division of Radiology, Faculty of Medicine and Surgery, University of Rome "Tor Vergata"

\section{REFERENCES}

1 FAO/WHO/UNU. Energy and protein requirements: technical report series 724. Geneva: World Health Organization, 1985.

2 McArdle WD, Katch Fl, Katch VL. Exercise physiology: energy, nutrition and human performance. 3rd ed. Philadelphia: Lea and Febiger, 1991.

3 Wilmore JH, Davis JA, Norton AC. An automated system for assessing metabolic and respiratory function during exercise. J Appl Physiol 1976;40:619-24

4 Consolazio CF, Johnson RE, Pecora L. Physiological measurements of metabolic functions in man. New York: McGraw-Hill Book Company, 1963.

5 Shepard RJ. A critical examination of the Douglas bag technique. J Physiol (Lond) 1955;127:515-24.

6 Macfarlane DJ. Automated metabolic gas analysis systems. A review. Sports Med 2001:31:841-61.

7 Lothian F, Farrally MR, Mahoney C. Validity and reliability of the Cosmed K2 to measure oxygen uptake. Can J Appl Physiol 1993; 18:197-206.

8 Crandall CG, Taylor SL, Raven PB. Evaluation of the Cosmed K2 portable telemetric oxygen uptake analyzer. Med Sci Sports Exerc 1994; 26:108-11. 
9 Lucia A, Fleck SJ, Gothshall RW, et al. Validity and reliability of the K2 Cosmed instrument. Int J Sports Med 1993;14:380-6.

10 Hausswirth C, Bigard AX, Le Chevalier JM. The Cosmed K4 telemetry system as an accurate device for oxygen uptake measurements during exercise. Int J Sports Med 1997; 18:449-53.

11 McLaughlin JE, King GA, Howley ET, et al. Validation of the K4 b2 portable metabolic system. Int J Sport Med 2001;22:280-4.

12 Pinnington $\mathrm{HC}$, Wong $\mathrm{P}$, Tay J, et al. The level of accuracy in measures of $\mathrm{FEO}_{2}, \mathrm{FECO}_{2}$ and VE between the Cosmed $\mathrm{K} 4 \mathrm{~b} 2$ portable, respiratory gas analysis system and a metabolic cart. J Sci Med Sport 2001;4:324-35

13 De Lorenzo A, Sorge RP, Bertini I, et al. Validity and reliability of a new portable telemetric calorimeter designed to measure oxygen consumption and carbon dioxide production. Diabetes Nutr Metab 2001;14:268-76.
14 Faina $M$, Pistelli R, Franzoso $G$, et al. Validity and realibility of a new telmetric portable system with $\mathrm{CO}_{2}$ analyzer (K4-Cosmed). Proceedings of the first annual congress of European College of Sport Science (Nice). 1996: 572-3.

15 Maldonado S, Mujika I, Padilla S. Influence of body mass and height on the energy cost of running in highly trained middle- and long-distance runners. Int J Sports Med 2002:23:268-72.

16 Gunn SM, Brooks AG, Withers RT, et al. Determining energy expenditure during some household and garden tasks. Med Sci Sports Exerc 2002;34:895-902.

17 Millet GY, Perrey S, Candau R, et al. Relationships between aerobic energy cost, performance and kinematic parameters in roller ski skating. Int J Sports Med 2002;23:191-5.

18 Burke LM. Energy needs of athletes. Can J Appl Physiol $2001 ; 26:$ S202-19.

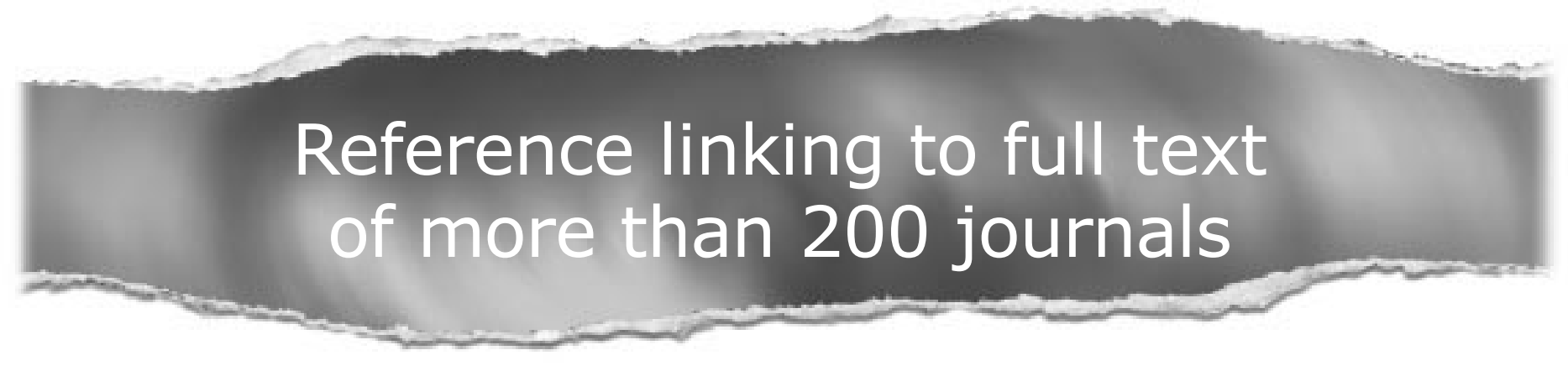

Toll free links

You can access the FULL TEXT of articles cited in the British Journal of Sports Medicine online if the citation is to one of the more than 200 journals hosted by HighWire (http://highwire.stanford.edu) without a subscription to that journal. There are also direct links from references to the Medline abstract for other titles.

www.bjsportmed.com 